\title{
Wave Packet Dynamics of Excited Atomic Electrons in Intense Laser Fields
}

\author{
K.C. Kulander \\ K.J. Schafer
}

This paper was prepared for submittal to the

15th International Conference on Atomic Physics

Amsterdam, Holland

August 5-9, 1996

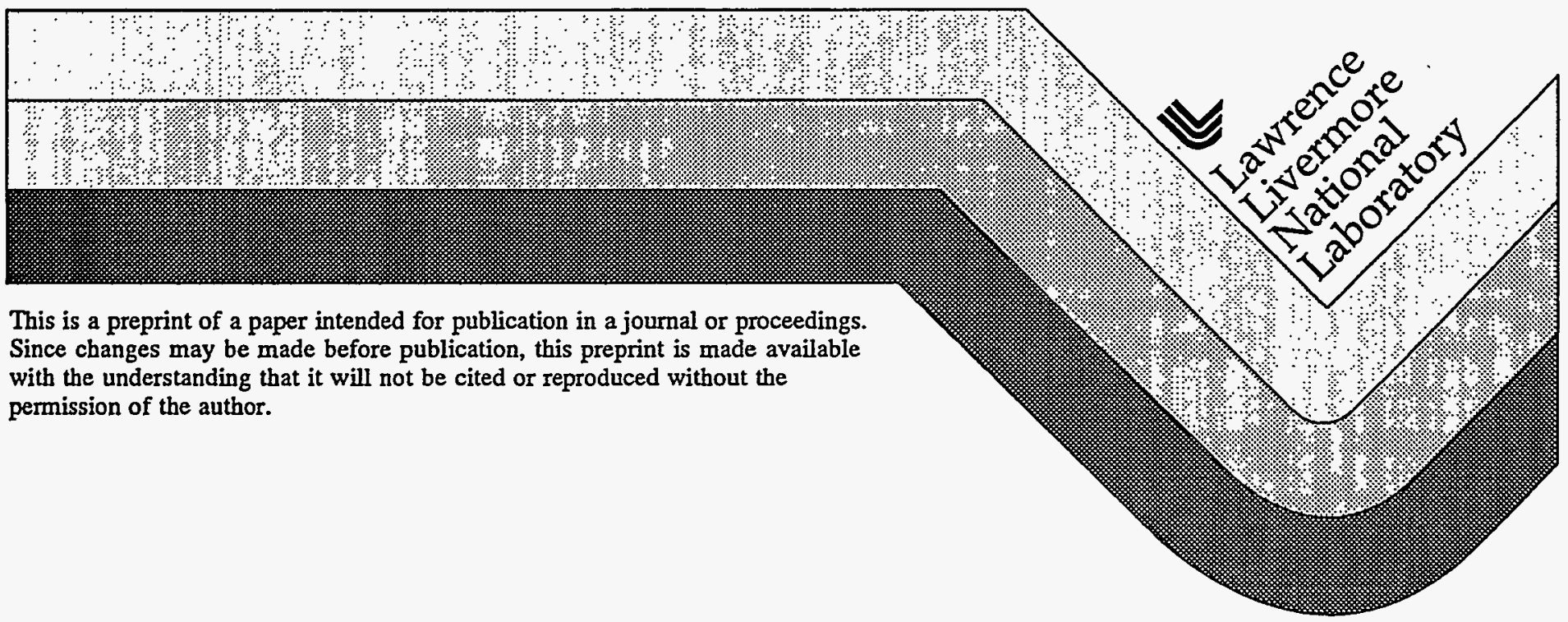




\section{DISCLAIMER}

This document was prepared as an account of work sponsored by an agency of the United States Government. Neither the United States Govermment nor the University of California nor any of their employees, makes any warranty, express or implied, or assumes anylegal liability or responsibility for the accuracy, completeness, or usefulness of any information, apparatus, product, or process disdosed, or represents that its use would notinfringe privately owned rights. Reference herein to any specific commercial products, process, or service by trade name, trademark, manufacturer, or otherwise, does not necessarily constitute or imply its endorsement, recommendation, or favoring by the United States Government or the University of California. The views and opinions of authors expressed herein do not necessarily state or rellect those of the United States Government or the University of California, and shall not be used for advertising or product endorsement purposes. 


\section{DISCLAIMER}

Portions of this document may be illegible in electronic image products. Images are produced from the best available original document. 


\title{
WAVE PACKET DYNAMICS OF EXCITED ATOMIC ELECTRONS IN INTENSE LASER FIELDS
}

\author{
K. C. KULANDER \\ TAMP Group, Physics Directorate, Lawrence Livermore National Laboratory, \\ Livermore CA 94551 USA \\ K. J. SCHAFER \\ Department of Physics and Astronomy, Louisiana State University, \\ Baton Rouge LA 70803 USA
}

\begin{abstract}
The dynamics of multiphoton ionization in the tunneling (long wavelength, high intensity) regime is described. Photoemission by tunnel ionized atoms is dominated by the odd harmonics of the driving laser field. Excitation by ultra short ( 10-20 fs) pulses produces high harmonics with characteristics which will allow them to be compressed to give colherent sources of VUV and possibly XUV radiation with pulse lengths near or below 1 fs.
\end{abstract}

\section{Introduction}

The ionization of rare gas atoms in strong ( $\mathrm{I}>10^{14} \mathrm{~W} / \mathrm{cm}^{2}$ ) visible and IR laser fields is dominated by the sequential stripping of one electron at a time. In this regime the ionized electron is freed from its ion core by tunneling through a potential barrier comprised of the Coulomb attraction of the screened nuclear charge and the instantaneous electric field of the laser. ${ }^{1,2,3}$ Tunneling will occur more rapidly near the two maxima in the electric field during each optical cycle. After escaping through the barrier the electron oscillates in response to the laser field as it drifts away from its ion core. The energy of oscillation can be quite large in comparison with the drift energy so that at least half the time the electron revisits the vicinity of the ion core before becoming completely free. During these collisions the electron may gain a substantial amount of drift energy, becoming a high energy photoelectron, or the electron may be recaptured into a bound state of the atom while simultaneously emitting a high energy photon. The resultant photoemission spectrum is dominated by frequencies which are integer multiples of the driving frequency, harmonic conversion. Because of the inversion symmetry of an atom, a linearly polarized laser field generates only odd harmonics. Thus a simple picture of ionization emerges in the strong-field, long wavelength regime: each half cycle a wave packet of electron probability escapes into the continuum via a tunneling process, then the wave packet drifts relatively slowly away from the ion, with a significant fraction of the density recolliding with ion core because of the large amplitude, oscillatory motion imposed by the driving field. 4,5,6 In this paper we will show how the observed characteristics of the excitation and emission processes can be easily interpreted in terms of this simple picture.

A major topic of current research focuses on the effects of using increasingly shorter, high intensity pulses on excitation and ionization processes. Pulse lengths as short as ten optical cycles (10s of fs) are becoming widely available. ${ }^{7,8}$ Changes in the observed harmonic emission spectrum generated using these ultra short pulses 
reflect modifications in the ionization dynamics that can be explained within the tunneling model. Here we will first present a discussion of the high order harmonic generation process including results obtained in longer pulse $(>1 \mathrm{ps})$ experiments. We then describe calculations of the emission spectrum for ultra short pulses and show that we can expect to exploit the short pulse harmonics to create VUV and XUV wavelength light sources with pulse lengths on the order of and possibly shorter than 1 fs.

\section{Harmonic Generation}

Because high order harmonic generation in a gas is a coherent process its efficiency. depends on both the emission from each individual atom and the propagation of the emitted fields through the excited volume as well, i.e., good phase matching is essential. ${ }^{9}$ Almost all experiments to date have been performed with tightly focused lasers to achieve high pump intensity. This focusing introduces a coherence length for the higher order harmonics which can be much shorter than the interaction length. This is alleviated to some degree in the high intensity regime by the non-perturbative nature of the single atom emission. It turns out that high order harmonics are produced over a much larger volume of the gas than would be expected based on perturbative calculations. ${ }^{10,11}$ This allows harmonic generation of very short wavelengths to be efficient enough to be a usable, coherent light source. In this paper we ignore phase matching effects to concentrate on the single atom emission for two reasons: (1) Most of the extremal properties of the full (single atom + phase matching) problem are constrained by the single atom behavior (in particular the minimum pulse length and maximum photon energy of the harmonics are so constrained), and (2) The advent of multi terrawatt lasers means that harmonic generation experiments with unfocused lasers are now possible, leading to simplified phase matching, at least for intensities below saturation (complete ionization) of the neutral gas atoms.

We calculate the single atom harmonic generation spectrum using the single active electron (SAE) approximation which has been extensively discussed in previous publications. ${ }^{12}$ Briefly, we solve the time-dependent Schrödinger equation (TDSE) for an atom in a linearly polarized laser pulse assuming that only the outermost valence electron responds to the field. Comparing our predictions using the SAE approximation to many different multiphoton experimental results has shown that for rare gas atoms and optical wavelengths this is generally a very good approximation. The SAE pseudopotential used in our calculations is obtained from a series of Hatree-Slater calculations for different angular momentum channels and results in very accurate excited state enerigies for rare gas atoms. We can numerically integrate the TDSE for the SAE to obtain its time-dependent wave function, $\psi$, which provides all the information about the various electron or photon emission processes. After choosing a laser wavelength, peak intensity and pulse envelope we calculate the emitted harmonic radiation by first calculating the dipole acceleration, $a(t)$, given by

$$
a(t)=\frac{d^{2}\langle z(t)\rangle}{d t^{2}}=-\langle\psi|H,[H, z]| \psi\rangle
$$


for each time step during the pulse. Here $H$ is the full (atomic + laser interaction) Hamiltonian. For the SAE pseudopotential, the commutator must be evaluated numerically. This form has been found to be computationally more stable than the usual dipole expression when significant ionization is present. ${ }^{13}$ The emitted radiation spectrum is proportional to the Fourier transform of the acceleration

$$
A(\omega)=\int d t e^{i \omega t} a(t) \equiv \mathcal{A}(\omega) e^{i \phi(\omega)}
$$

where $\mathcal{A}(\omega)$ is the (real) spectral envelope function. The acceleration spectrum $A(\omega)$ is equal to $\omega^{2} D(\omega)$, where $D(\omega)$ is defined to be the "dipole spectrum" obtained by Fourier transforming $\langle z(t)\rangle$. We can also calculate an approximate $D(\omega)$ as outlined in Ref. 13 by taking into account only transitions back to the ground state and find that it agrees with $A(\omega) / \omega^{2}$ for all of the harmonics in the plateau and cutoff. This clearly demonstrates that the harmonics are produced only by excited electrons which return to the immediate vicinity of the ion core.

We next present some of the basic features of high order harmonics, including the effects of using shorter pulses on the spectral structure and maximum harmonic order. Following that we demonstrate that the harmonics created at the peak of a short laser pulse can be compressed using a VUV grating pair arranged to provide positive group velocity dispersion to give pulses approaching the attosecond regime.

\subsection{Single-Atom Spectra}

At long wavelength and high intensity harmonic generation involves the excitation of electrons into the continuum and rescattering from the parent ion. $4,5,6$ This implies a close relationship between harmonic generation and ionization. In particular the highest order harmonic that is emitted is limited by the highest intensity that any atom experiences before ionizing in accord with the cutoff rule $E_{\max } \sim I P+3 U_{p}$ where $I P$ is the ionization potential and $U_{p}$ the intensity-dependent ponderomotive energy. ${ }^{14}$ In Fig. 1 we show the spectrum generated by neon when excited by a "long" pulse $1064 \mathrm{~nm}$ laser at an intensity of $6 \times 10^{14} \mathrm{~W} / \mathrm{cm}^{2}$. By long pulse we mean one in which the envelope of the pulse varies slowly compared to the laser frequency ( $>1$ ps.) The spectrum consists of narrow peaks at odd multiples of the fundamental which have approximately constant strength (the plateau) up to a maximum harmonic order predicted by the cutoff rule (arrow). The width of the individual harmonic lines is determined by the shorter of the pulse width or the ionization time. As shown in Eq. (2), these harmonics will have a phase, $\phi(\omega)$, relative to the driving field. In the long pulse case these phases vary rapidly with intensity and almost randomly, modulo $2 \pi$, from one harmonic to the next. We note that the SAE emission spectra agree well with those observed in the many recent experimental studies of high order harmonic generation.

By contrast, the emission spectrum from an atom in an ultra short pulse is very different. This is demonstrated in Fig. 2 where the high end of the "harmonic" spectrum from argon in a $27 \mathrm{fs}, 810 \mathrm{~nm}$ pulse is displayed in the upper plot and the corresponding relative frequency-dependent phase is shown in the lower plot. First we note that the cutoff rule still holds, predicting the end of the plateau 


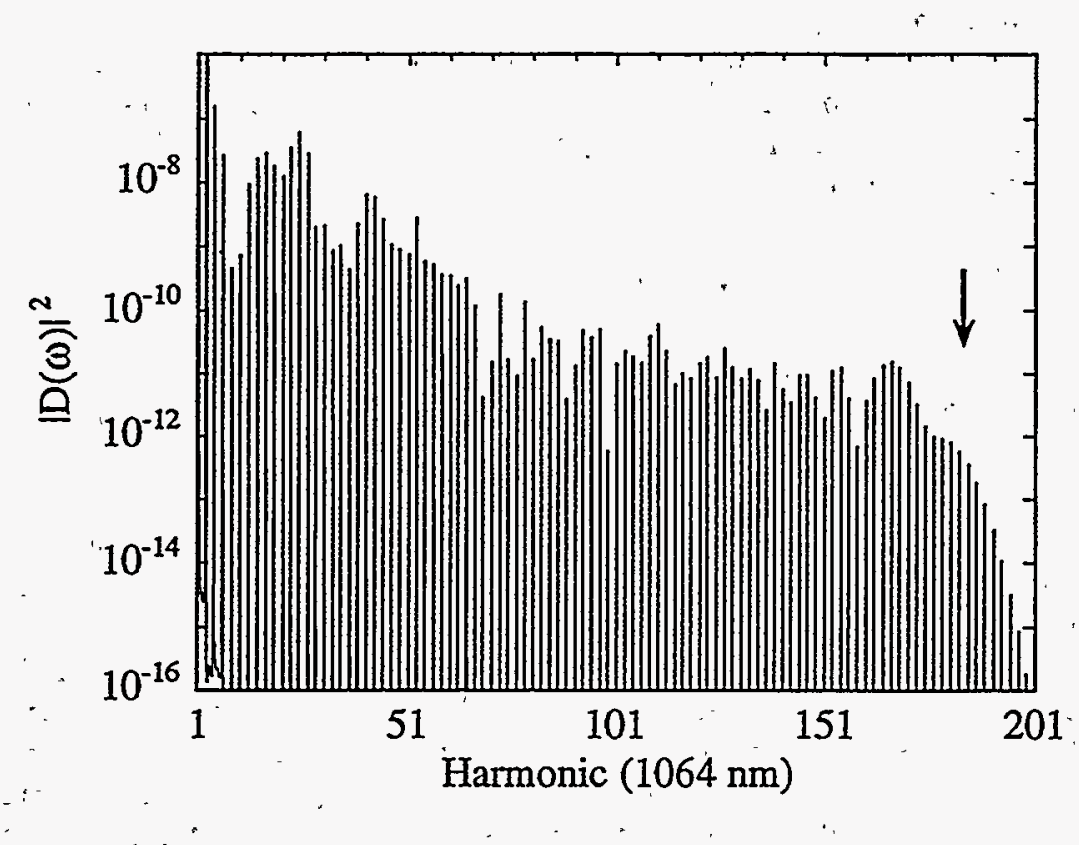

Figure 1: Harmonic emission spectrum from neon excited by a $1064 \mathrm{~nm}$ long pulse laser at an intensity of $6 \times 10^{14} \mathrm{~W} / \mathrm{cm}^{2}$.

to be around the 47 th harmonic. We find that only the last few harmonics are distinct and they are very broad compared to the long pulse case. In fact they are even broader than one would expect from this very short pump pulse, i.e., $k^{2} \Delta \lambda_{k} \gg \Delta \lambda_{1}$. Here $k$ is the order of the harmonic and $\lambda_{1}$ is the wavelength of the pump. Surprising also is that the phases of these highest harmonics are well behaved. The spectrum below the vicinity of the cutoff, where the plateau harmonics are expected, is highly structured with the odd harmonic peaks being hard to distinguish and their frequency-dependent relative phases varying rapidly. We find this. very different spectrum to be typical in the ultra short pulse regime.

The mechanism for producing spectra such as that shown in Fig. 2 becomes clear when we consider the ionization dynamics of the tunneling model described above. The $27 \mathrm{fs}$ pulse has a half-width of ten optical cycles at this frequency. This means that during both the rising and falling parts of the pulse, the laser intensity is changing significantly on the time scale of a single cycle. Consider a tunneling wave packet created in one half cycle during the pulse rise. The field which turns the escaping wave packet around to rescatter from the ion core one half cycle later is stronger than it was when the wave packet was created. Therefore the recollision velocity is higher than the one encountered in a constant intensity field. Also the time between when the wave packet is created and its return is shorter when the intensity is rapidly rising. Both these effects cause a blue shift in the "harmonic" frequency. Similarly, on the falling edge of the pulse, a red shift occurs. Only those harmonics produced within a short time interval near the peak of the pulse will be 


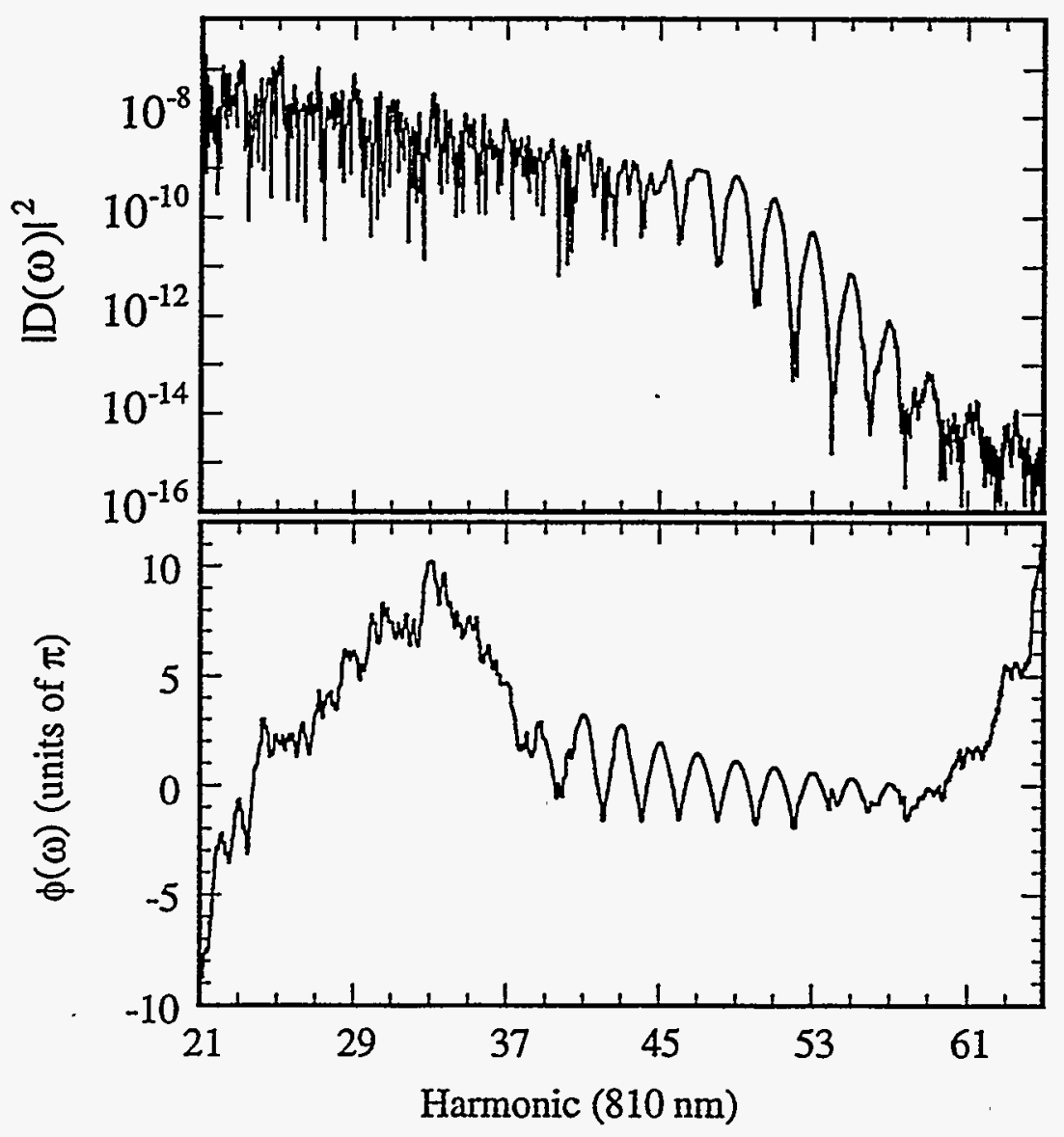

Figure 2: Harmonic emission spectrum (upper plot) from argon excited by a $810 \mathrm{~nm}, 27 \mathrm{fs}$ pulse at an intensity of $3 \times 10^{14} \mathrm{~W} / \mathrm{cm}^{2}$. The lower plot shows the phases of the emitted radiation, $\phi(\omega)$, defined in Eq. (2). 


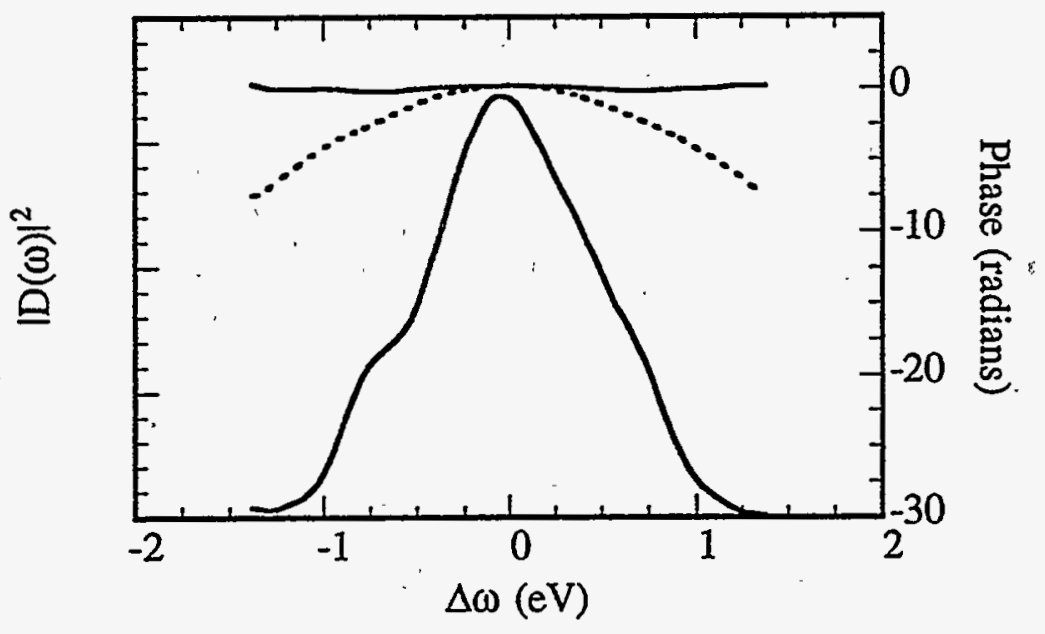

Figure 3: Emission spectrum in the vicinity of the 49th harmonic from argon excited by a $810 \mathrm{~nm}$, 27 fs pulse at an intensity of $3 \times 10^{11} \mathrm{~W} / \mathrm{cm}^{2}$. The dotted line shows the phases of the emitted radiation and the upper solid line shows the phase with its quadratic component removed.

unshifted. According to the cutoff rule, there is a well defined relationship between the laser intensity and the maximum harmonic produced. For the harmonics within the plateau, the intensity is high enough for them to be generated during a large fraction of the pulse. These are emitted, with a blue shift, as the pulse rises and with a red shift as the intensity declines. The resulting spectrum at these mid-plateau harmonic wavelengths is broadened to the extent that the peaks have disappeared and the emission strength shows substantial interference. On the other hand the rule indicates that those harmonics at the end of the plateau are produced only at the peak of the pulse. The short time interval during which they are emitted minimizes any interference. From the discussion above we would still expect these cutoff harmonics to have a linear chirp, which is equivalent to a quadratic phase change, if they are produced just as the pulse passes through its maximum. This is exactly what is found as indicated in Fig. 2. We show this in more detail in Fig. 3 where the emission strength and the phase for the 49th harmonic are plotted. The phase shift, shown by the dotted line, is clearly parabolic. The amplitude of the harmonic is peaked almost exactly at the expected, unshifted line center. These unexpected properties of the cutoff harmonics turn out to be ideal for producing pulses of VUV and XUV radiation which are more than an order of magnitude shorter than the driving pulse width, and, in fact, even shorter than an optical cycle of the incident field. 


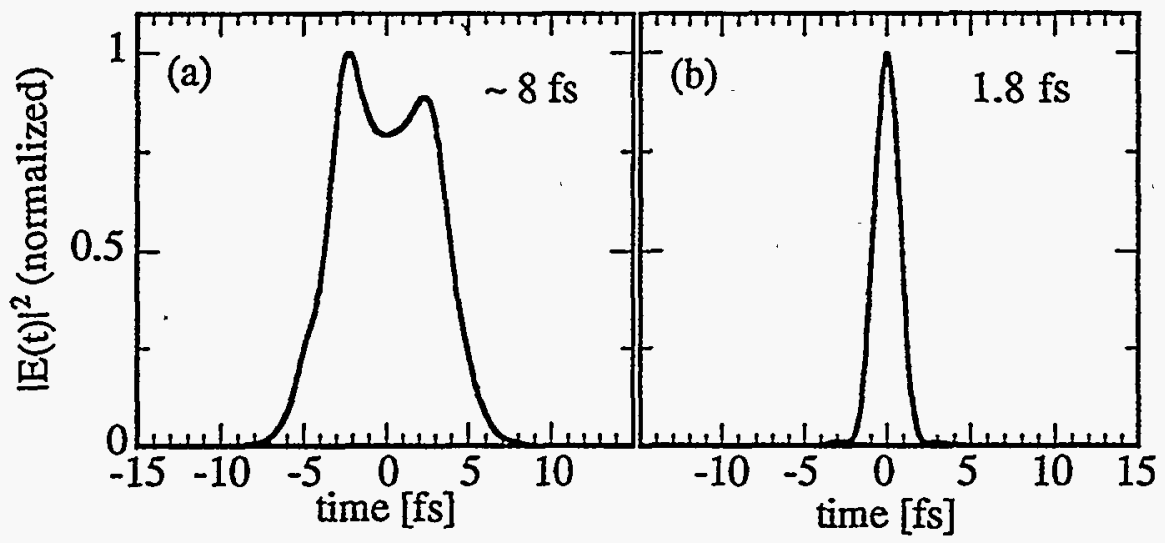

Figure 4: The (a) raw and (b) compressed envelope of the 49th harmonic from argon excited by an $810 \mathrm{~nm}, 27 \mathrm{fs}$ pulse at an intensity of $3 \times 10^{14} \mathrm{~W} / \mathrm{cm}^{2}$.

\subsection{Compressing The Cutoff Harmonics}

To calculate the pulse width of the $k$ th harmonic we examine the emitted electric field intensity envelope. This is obtained by back transforming the acceleration spectrum after applying a filter function around the frequency $\omega_{k}$ :

$$
\mathcal{E}(t)=e^{-i \omega_{k} t} \int d \omega e^{-i\left(\omega-\omega_{k}\right) t}\left[\mathcal{A}(\omega) e^{i \phi(\omega)} F\left(\left(\omega-\omega_{k}\right)\right] \equiv E_{k}(t) e^{-i\left[\omega_{k} t-\phi_{k}(t)\right]}\right.
$$

$F\left(\omega-\omega_{k}\right)$ is a square filter that roughly duplicates the action of a grating in picking out a specific harmonic. As an example we again focus on the 49 th harmonic $(\sim 75$ $\mathrm{eV}$ ) in Fig. 3. Since the energy falls by several orders of magnitude before and after the 49th harmonic the shape of the filter function used is not crucial. The raw phase of this harmonic (dotted line in Fig. 3) is almost purely quadratic in the detuning $\Delta \omega=\omega_{49}-\omega$. The phase minus its quadratic component

$$
\tilde{\phi}(\omega)=\phi(\omega)-0.5 \times\left.\left(\omega_{49}-\omega\right)^{2} \frac{d^{2} \phi}{d \omega^{2}}\right|_{\omega=\omega_{k}}
$$

is shown as a solid line above the envelope in Fig. 3. The calculated time history is displayed in Fig. 4a which clearly shows that this harmonic is emitted only near the peak of the pulse. A similar calculation for a mid-plateau harmonic would show a much longer time of emission and considerable structure because of interference. The emission envelope for the 49th harmonic shows a small asymmetry with the first peak being slightly stronger than the peak which appears just after the maximum in the laser pulse. Since the early time contributions to the harmonic are blue shifted, we expect and see that the blue wing of the harmonic (Fir. 3) is slightly stronger.

The linear chirp in this harmonic can be entirely removed using a grating pair by introducing a positive group velocity dispersion which exactly compensates for 
the quadratic component of the phase. ${ }^{15} \mathrm{We}$ can mimic this by replacing $\phi(\omega)$ by $\tilde{\phi}(\omega)$ in Eq. (3) and recalculating the pulse envelope. The compressed spectrum is shown in Fig. $4 \mathrm{~b}$. Its width, $1.8 \mathrm{fs}$, is within a few percent of the transform limit for the spectral envelope function. This is because the phase becomes almost constant when the quadratic term is removed as can be seen in Fig. 3. This pulse width is a factor of 15 smaller than the incident laser pulse and shorter than the 2.7 fs period of the driving field. The peak heights in Fig. 4 have been normalized to one for the purposes of plotting. In reality the energy of the pulse is conserved, leading to a much higher peak field in the compressed case.

We have repeated the calculations illustrated in Figs. 2-4 for a range of peak intensities in argon and neon at $810 \mathrm{~nm}$. Qualitatively the results are the same. The harmonics, at the end of the plateau exhibit a simple linear chirp which can be removed to provide $<2$ fs pulses from a single harmonic. The highest efficiency is obtained by compressing harmonics that are at the end of the plateau for peak intensities just below the saturation intensity. Above that intensity interferences destroy the simple quadratic phase dependence. Using neon, with its higher ionization potential, instead of argon allows us to reach higher photon energies.

In addition, as is clear from Fig. 2, several of the harmonics near the cutoff have almost the same group delay $(d \phi / d \omega)$. This is a general feature in all of the short pulse harmonics calculations that we have carried out. In principle the same compressor that can produce femitosecond pulses from a single harmonic can be used to produce sub-femtosecond pulses by combining several harmonics. Removing the average group delay of three cutoff harmonics and back transforming yields an intensity profile similar to that shown in Fig. $4 \mathrm{~b} .{ }^{15}$ However, in this case the width of the central feature is found to be as short as $\sim 400$ attoseconds. This seems to be one of the most promising ways to reach the sub-femtosecond regime.

\section{Conclusion}

We have presented the simple picture which has recently emerged of the dynamics of strong field multiphoton ionization of atoms. The electrons escape from the atom by tunneling through an instantaneous suppressed barrier. Each half cycle of the field a wave packet is promoted into the continuum and its subsequent evolution is determined predominantly by its interaction with the oscillating electric field of the laser. The driven oscillations of the wave packet cause additional encounters with the parent ion core resulting in the surprisingly efficient generation high order harmonics. If the driving field is an ultra short pulse, the wave packet dynamics favors the production of very well defined but broad harmonic peaks for the highest energy members of the plateau. These harmonics are created with a linear chirp which can be removed to yield pulses as short as $\sim 1-2 \mathrm{fs}$. If harmonics from several neighboring peaks are combined, the pulse lengths can be pushed down into the attosecond regime. 


\section{Acknowledgments}

This work has been carried out in part under the auspices of the U.S. Department of Energy at the Lawrence Livermore National Laboratory under contract No. W7405-ENG-48.

\section{References}

1. H. B. van Linden van den Heuvell and H. G. Muller, in Multiphoton Processes, eds. S. Smith and P. L. Knight (Cambridge University Press, Cambridge, 1988).

2. T. F. Gallagher, Phys. Rev. Lett. 61, 2304 (1988).

3. P. B. Corkum, N. H. Burnett and F. Brunel Phys. Rev. Lett. 62, 1259 (1989).

4. K. J. Schafer, B. Yang, L. F. DiMauro and K. C. Kulander, Phys. Rev. Lett. 70, 1599 (1993).

5. K. C. Kulander, K. J. Schafer and J. I. Krause, in Super-Intense Laser-Atom Physics, eds. B. Pireaux, A. L'Huillier and K. Rzazewski (Plenum, New York, 1993).

6. P. B. Corkum, Phys. Rev. Lett. 71, 1994 (1993).

7. J. Zhou, J. Peatross, M. M. Murnane, H. C. Kapteyn and I. P. Christof, Plys. Rev. Lett. 761, 752 (1996).

8. C. P. J. Barty et al., Opt. Lett. 21, 668 (1996).

9. A. L'Huillier, K. J. Schafer and K. C. Kulander, J. Phys. B 24, 315 (1991).

10. A. L'Huillier, K. J. Schafer and K. C. Kulander, Phys. Rev. Lett. 66, 2200 (1991).

11. A. L'Huillier, P. Balcou, S. Candel, K. J. Schafer and K. C. Kulander, Phys. Rev. A 46, 2778 (1992).

12. K. C. Kulander, K. J. Schafer and J. L. Krause, in Atoms in Intense Laser Fields, ed. M. Gavrila (Academic Press, New York, 1992).

13. J. L. Krause, K. J. Schafer and K. C. Kulander, Pliys. Rev. A 45, 4998 (1992).

14. J. L. Krause, K. J. Schafer and K. C. Kulander, Phys. Rev. Lett. 68, 3535 (1992).

15. K. J. Schafer, K. C. Kulander, J. A. Squire and C. P. J. Barty, in Generation, amplification and measurement of ultra short pulses, III, SPIE 2701, (1996) in press. 